\title{
Unsteady Simulation of a Synthetic Jet Actuator with Cylindrical Cavity Using a 3-D Lattice Boltzmann Method
}

\author{
Hongbin Mu $\mathbb{D},{ }^{1,2}$ Qingdong Yan, ${ }^{1}$ Wei Wei, ${ }^{1}$ and Pierre E. Sullivan $\mathbb{D}^{2}$ \\ ${ }^{1}$ School of Mechanical Engineering, Beijing Institute of Technology, Beijing, China \\ ${ }^{2}$ University of Toronto, Department of Mechanical and Industrial Engineering, Toronto, ON, Canada \\ Correspondence should be addressed to Hongbin Mu; hongbin.mu@mail.utoronto.ca
}

Received 28 August 2017; Revised 15 December 2017; Accepted 25 December 2017; Published 11 April 2018

Academic Editor: Vaios Lappas

Copyright (C) 2018 Hongbin Mu et al. This is an open access article distributed under the Creative Commons Attribution License, which permits unrestricted use, distribution, and reproduction in any medium, provided the original work is properly cited.

\begin{abstract}
A synthetic jet actuator is a zero-net mass-flux device that imparts momentum to its surroundings and has proved to be a useful active flow control device. Using the lattice Boltzmann method (LBM) with the Bhatnagar-Gross-Krook (BGK) collision models, a 3-D simulation of a synthetic jet with cylindrical cavity employing a sinusoidal velocity inlet boundary condition was conducted. The velocity distributions are illustrated and discussed, and the numerical results are validated against previous experimental data. The computed results show the ingestion and expulsion flow over one working cycle as well as the evolution of vortices important to the control of the separated shear layer. Zero-net mass-flux behavior is confirmed.
\end{abstract}

\section{Introduction}

A synthetic jet transfers linear momentum to the surroundings by alternately ingesting and expelling fluid from a cavity containing an oscillating diaphragm and has proven to be a useful active flow control device [1-3]. Compared with continuous jets, synthetic jets are low-weight, compact devices and do not require internal fluid supply lines [4-6]. A schematic diagram of a typical synthetic jet actuator is shown in Figure 1.

A synthetic jet actuator has a nozzle or slot connected to a cavity in which typically a piezoelectric diaphragm oscillates. By oscillating the diaphragm, the working fluid is alternately ingested and expelled through the nozzle exit, forming a train of discrete vortical structures that convert linear momentum to flow without net mass injection [7]. The fact that no external fluid source is required combined with the availability of increasingly small vibrating diaphragms, for example, piezoelectric disks, allows the design of extremely compact devices, even down to MEMS scale [8-10].

Okada et al. [11] studied the influence of amplitude and frequency of a synthetic jet using large-eddy simulation (LES) and concluded that three-dimensional modeling of the flow inside the cavity was essential for accurate estimation of the velocity and velocity fluctuations of the synthetic jet. Ma et al. [12] investigated the influences of periodic velocity inlet and dynamic mesh boundary conditions for synthetic jet simulation, and two cases with different boundary conditions were conducted, respectively, at low Reynolds number $(\mathrm{Re}=10.7)$ with the Computational Fluid Dynamics(CFD) method. Utturkar et al. [13] used an incompressible flow model to study the flow in rectangular cavities where the aspect ratio and location of the oscillating diaphragm was varied. Cavities containing multiple oscillating diaphragms were also considered, where the oscillation amplitude was adjusted in order to maintain the same volume displacement in each case. Feero et al. [14] presented the first experimental effort to validate the limited number of numerical investigations that have postulated that synthetic jets are insensitive to cavity shape. Three axisymmetric synthetic jets with different cavity shapes were used to examine jet performance while keeping other parameters constant such as cavity volume, nozzle length, and nozzle diameter. The experimental results showed that synthetic jet performance is dependent on cavity shape.

The lattice Boltzmann method (LBM) offers an alternative method to simulate complex fluid flow, allowing easy implementation of boundary conditions and can be used 


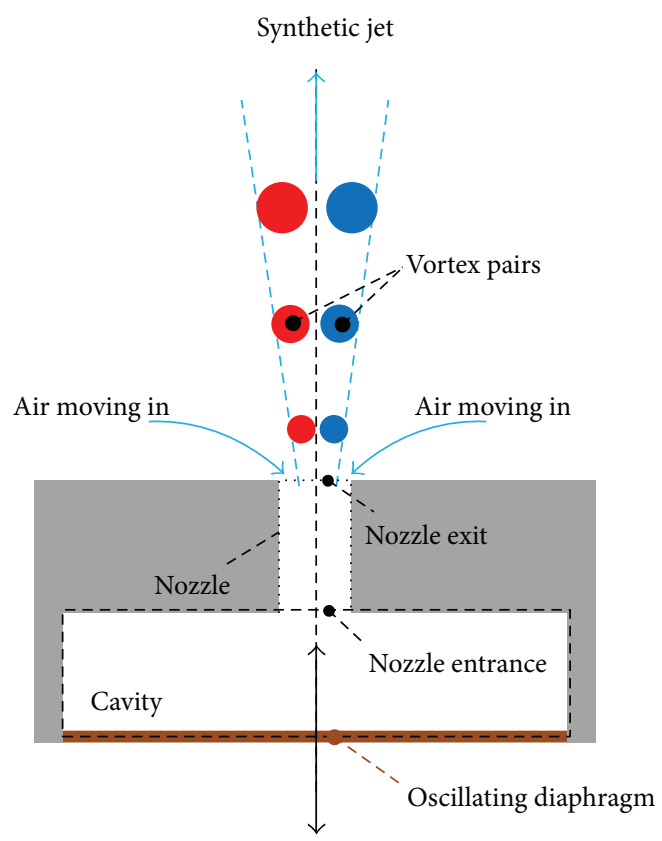

FIgURE 1: Schematic diagram of a synthetic jet actuator.

for unsteady flows, phase separation, evaporation, condensation, cavitation, solute and heat transport, and buoyancy, and interactions with surfaces can readily be simulated. Instead of solving the classical macroscopic Navier-Stokes (N-S) equations, as is done with traditional CFD methods, LBM solves for a limited number of interactions with small particles to determine the exchange of momentum and energy by simulating streaming and collision processes [15]. The LBM is based on constructing simplified kinetic models containing the physics of microscopic and mesoscopic processes so that averaging can recover macroscopic properties that obeys the continuum equations [16].

LBM also has been applied to flow control including flow separation control, mixing control, and turbulence control, using a pair of synthetic jets (SJs). Wang et al. [17] studied the active control of wakes and one-dimensional vortexinduced vibrations (VIVs) from a single circular cylinder using a pair of synthetic jets (SJs) at a low Reynolds number, $\mathrm{Re}=100$, using a lattice Boltzmann method. With a hybrid approach of the lattice Boltzmann method for flow field computations and a finite difference, Mautner [18] studied the modified main channel flow results for various wall jet geometries (derived from synthetic jets), jet inlet conditions, scaling issues, and Reynolds numbers. Fu et al. [19] reported on the use of the lattice Boltzmann method, in conjunction with large-eddy simulations, to study an interesting phenomenon related to the suppression of vortex shedding from circular cylinders with high accuracy coupled with computational efficiency. It had been observed in experiments that vortex shedding and the separated shear layer from a cylinder could be drastically reduced by the injection of a fluid jet into the approach flow.

The application of LBM for fluid flow simulations is far less common than finite volume methods; however, there is sufficient literature that suggests this method is accurate and less computationally intensive than others. For this work, a 3-D simulation of a synthetic jet cavity was conducted with the lattice Boltzmann method which allowed more accurate and comprehensive performance data for synthetic jet flow than previous 2-D simulations. A 3-D nondimensional numerical model of synthetic jet actuator was employed to simulate this synthetic jet. The D3Q19 model (Figure 2) was used for this work and consists of 19 distribution functions [20].

The goal of the present study is to study synthetic jet performance with a cylindrical cavity using 3-D lattice Boltzmann unsteady simulation. The numerical simulation was validated using previous experimental data [14]. This was extended to show the behavior of the synthetic jet actuator in quiescent flow.

\section{Numerical Method}

A synthetic jet actuator was modeled with an axisymmetric cylindrical cavity as shown in Figure 3(a). A 2-D view of the 3-D computational domains is shown in Figure 3(b). The different sections shown in Figure 3(b) represent the different boundary conditions where the red line is the inlet velocity profile, the black shows the no-slip wall, and the blue shows the outlet with outflow condition.

The nozzle diameter $d=2 \mathrm{~mm}$ and nozzle length $l_{\mathrm{n}}=10 \mathrm{~mm}$ were held constant. A nozzle diameter of $2 \mathrm{~mm}$ is determined to be the minimum size where adequate spatial resolution should be achieved in the $3-\mathrm{D}$ simulation across the nozzle. The cavity diameter is $D_{c}=12 \mathrm{~mm}$, and the cavity length is $l_{c}=10 \mathrm{~mm}$. The size of the exterior region is $l_{\mathrm{e}} \times w_{\mathrm{e}} \times w_{\mathrm{e}}$ or $40 \mathrm{~mm} \times 16 \mathrm{~mm} \times 16 \mathrm{~mm}$. The frequency $f$ of the diaphragm sinusoidal oscillation velocity $U_{\text {dia }}$ is given by

$$
U_{\text {dia }}=U_{\text {dia } \_ \text {max }} \sin (2 \pi f t),
$$

where $U_{\text {dia_max }}$ is the magnitude of the maximum diaphragm velocity at the cavity inlet. This boundary condition provides a simple sinusoidal representation of the mass flux produced in the nozzle by the motion of the diaphragm.

The characteristic velocity of a synthetic jet is often defined as the axial centerline velocity, $U_{c l}$, due to the assumption of a simple top-hat velocity profile at the nozzle/slot exit and was used to allow matching with the companion experiments. However, since the spatial velocity profile can show significant deviation from the top-hat shape, a more general velocity scale is the time-and-spatially averaged exit velocity over the expulsion stroke as follows:

$$
U_{\text {ave_ts }}=\frac{2}{T} \frac{1}{A_{\mathrm{n}}} \int_{A_{\mathrm{n}}} \int_{0}^{T / 2} U\left(t, A_{\mathrm{n}}\right) d t d A_{\mathrm{n}},
$$

where $U$ is the phase-averaged axial jet velocity at the nozzle exit plane, $T$ is the period, and $A_{n}$ is the nozzle exit area. The average velocity on the centerline is used to define a jet Reynolds number, given by

$$
\operatorname{Re}_{U_{\text {ave_ts }}}=\frac{U_{\text {ave_ts }} d}{v},
$$




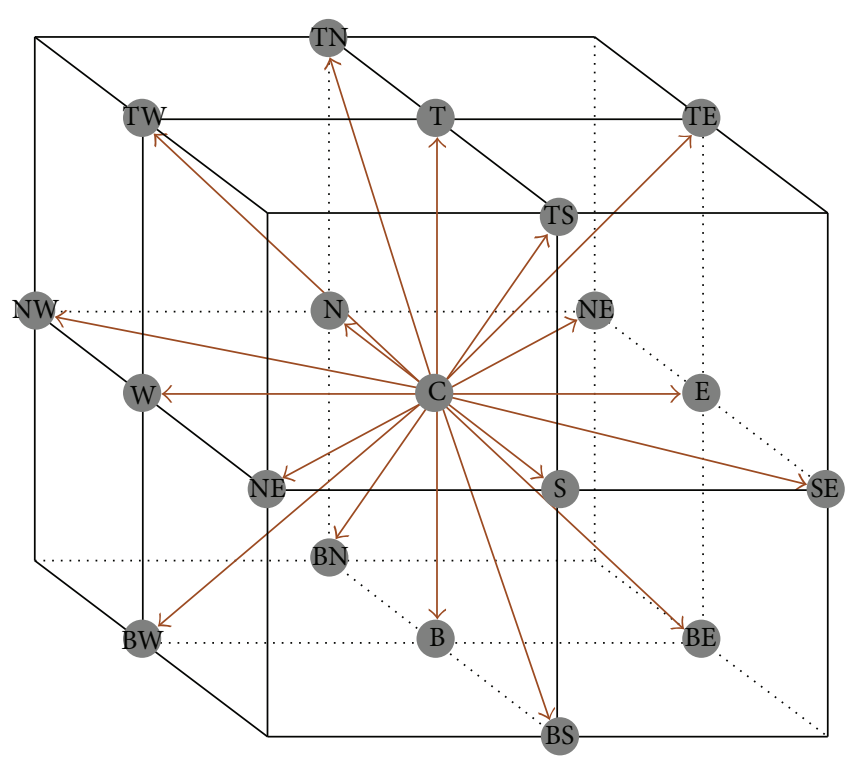

Figure 2: D3Q19 model for the 3-D LBM.

where $v$ is the kinematic viscosity of the working fluid, $14.8 \times 10^{-6} \mathrm{~m}^{2} / \mathrm{s}$, and $d$ is the slot width or nozzle diameter. The important length scale in synthetic jet flow is the nondimensional stroke length $L_{0} / d$, where

$$
L_{0}=\int_{0}^{T / 2} U_{\text {ave_s }}(t) d t
$$

where $U_{\text {ave_s }}$ is the spatially averaged velocity at the nozzle exit. The stroke length represents the distance that the fluid travels during the expulsion portion of the cycle. The excitation frequency $f$ can be nondimensionalized as a Stokes number, $S$ :

$$
S=\sqrt{\frac{2 \pi f d^{2}}{v}} .
$$

These nondimensional parameters define the operating conditions of a synthetic jet and greatly influence its ability to transfer linear momentum. Holman et al. [21] showed that in order to achieve vortex escape after expulsion, a synthetic jet formation value must exceed

$$
\frac{\operatorname{Re}_{U_{\text {ave-ts }}}}{S^{2}}>C \text {, }
$$

where $C$ is a constant equal to 0.16 for $3-\mathrm{D}$ nozzles. $\operatorname{Re}_{U_{\text {ave } t \mathrm{ts}}}=676 . S$ is 22 . When $S$ increases to approximately 20 , the average velocity reaches $90 \%$ of the centerline velocity and continues to asymptote towards $100 \%$ with increasing $S . U_{\text {ave_smax }}$ is the amplitude of the spatially averaged velocity $U_{\text {ave_s }}$ in the expulsion stroke, namely the maximum $U_{\text {ave } \_}$, which could be calculated as [21].

$$
U_{\text {ave_ts }}=\frac{2}{\pi} U_{\text {ave_smax }} \text {. }
$$

To meet the formation criteria for a synthetic jet actuator [20], $\operatorname{Re}_{U_{\text {ave-ts }}} / S^{2}>C, C=0.16$, so $f<U_{\text {ave_ts }} /(2 \pi d C)$, $f<2488 \mathrm{~Hz}$. In this work, $f$ is set as $300 \mathrm{~Hz}$ and $\operatorname{Re}_{U_{\text {ave }} / S^{2}}$ $=1.41$, which is one of the study cases of Feero et al. [14] meeting the formation criterion for synthetic jets.

As for the present 3-D numerical model, the $U_{\text {dia_max }}$ could be obtained through mass conservation [22]:

$$
U_{\text {dia_max }}=\frac{d^{2}}{D^{2}} U_{\text {ave_smax }}=\frac{\pi d^{2}}{2 D^{2}} U_{\text {ave_ts }} .
$$

Given the above, $U_{\text {dia_max }}$ could be obtained as $0.5 \mathrm{~m} / \mathrm{s}$, and $U_{\text {dia }}=[0.5 \sin (600 \pi t)] \mathrm{m} / \mathrm{s}$.

The velocity at the cavity inlet $U_{\text {dia_pmax }}$ should be

$$
U_{\text {dia_p } \max }=U_{\text {dia_max }} \text {. }
$$

Here, $U_{\text {dia_pmax }}$ is set as $0.5 \mathrm{~m} / \mathrm{s}$, the diaphragm velocity $U_{\text {dia_LBmax }}$ in lattice unit is $0.005 \mathrm{u}$, and the resolution of the reference length $N$ is 100 . The total lattice scale domain was $2000 \times 800 \times 800$ for accurate results. The physical length $L_{\mathrm{p}}$ is $2 \mathrm{~mm}$, equal to the value of $d$, so the grid spacing and time step could be obtained as follows:

(1) $\Delta x$, grid spacing for one lattice length,

$$
\Delta x=\frac{L_{\mathrm{p}}}{N}
$$

(2) $\Delta t$, time step for one lattice time,

$$
\Delta t=\frac{L_{\mathrm{p}} \cdot U_{\text {dia_LB max }}}{N \cdot U_{\text {dia_p } \max }}
$$
follows:

The physical velocity at the cavity inlet $U_{\text {dia } \_ \text {p }}$ is as

$$
U_{\text {dia_p }}=U_{\text {dia_pmax }} \sin \left(2 \pi f_{\mathrm{p}} t_{\mathrm{p}}\right),
$$

where $f_{\mathrm{p}}$, the physical frequency of diaphragm velocity, is $300 \mathrm{~Hz}$, and the physical period $T_{\mathrm{p}}$ is about $0.0033 \mathrm{~s}$, and $t_{\mathrm{p}}$ is the physical time. Thus, the diaphragm velocity in lattice unit is

$$
\begin{aligned}
f_{\mathrm{LB}} & =f_{\mathrm{p}} \Delta t, \\
t_{\mathrm{LB}} & =\frac{t_{\mathrm{p}}}{\Delta t}, \\
U_{\text {dia } \_ \text {LB }} & =U_{\text {dia_LBmax }} \sin \left(2 \pi f_{\mathrm{LB}} t_{\mathrm{LB}}\right),
\end{aligned}
$$

where $f_{\mathrm{LB}}$ is the frequency of diaphragm velocity in lattice unit and $t_{\mathrm{LB}}$ is the time in lattice unit.

\section{Results and Discussion}

The physical fluxes at the diaphragm (location of the piezoelectric membrane in the experiments) and nozzle exit, $Q_{\text {dia }}$ and $Q_{\text {nozzle exit, }}$ were modeled and are shown in Figure 4(a). The flux at different times was normalized by its corresponding magnitude in working cycles, so the 


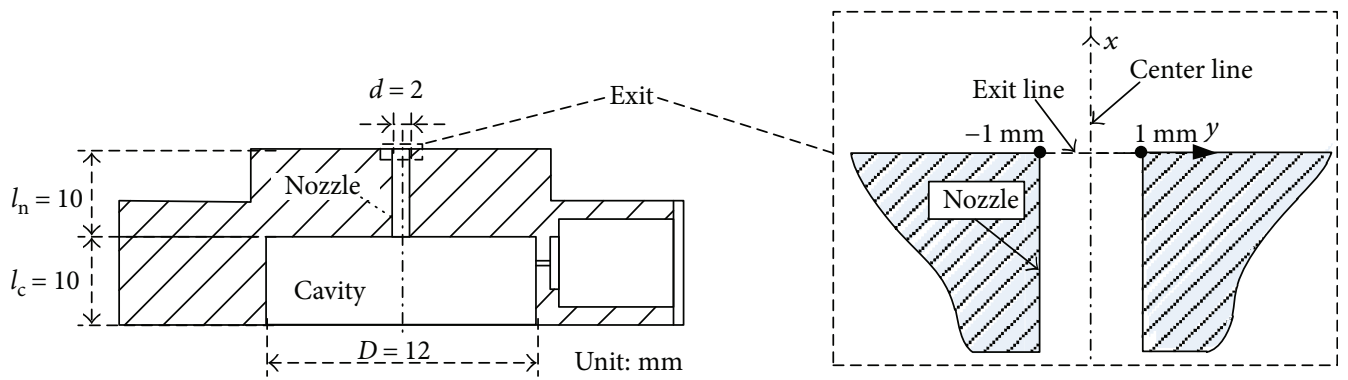

(a) Cylindrical axisymmetric synthetic jet cavity cross section A
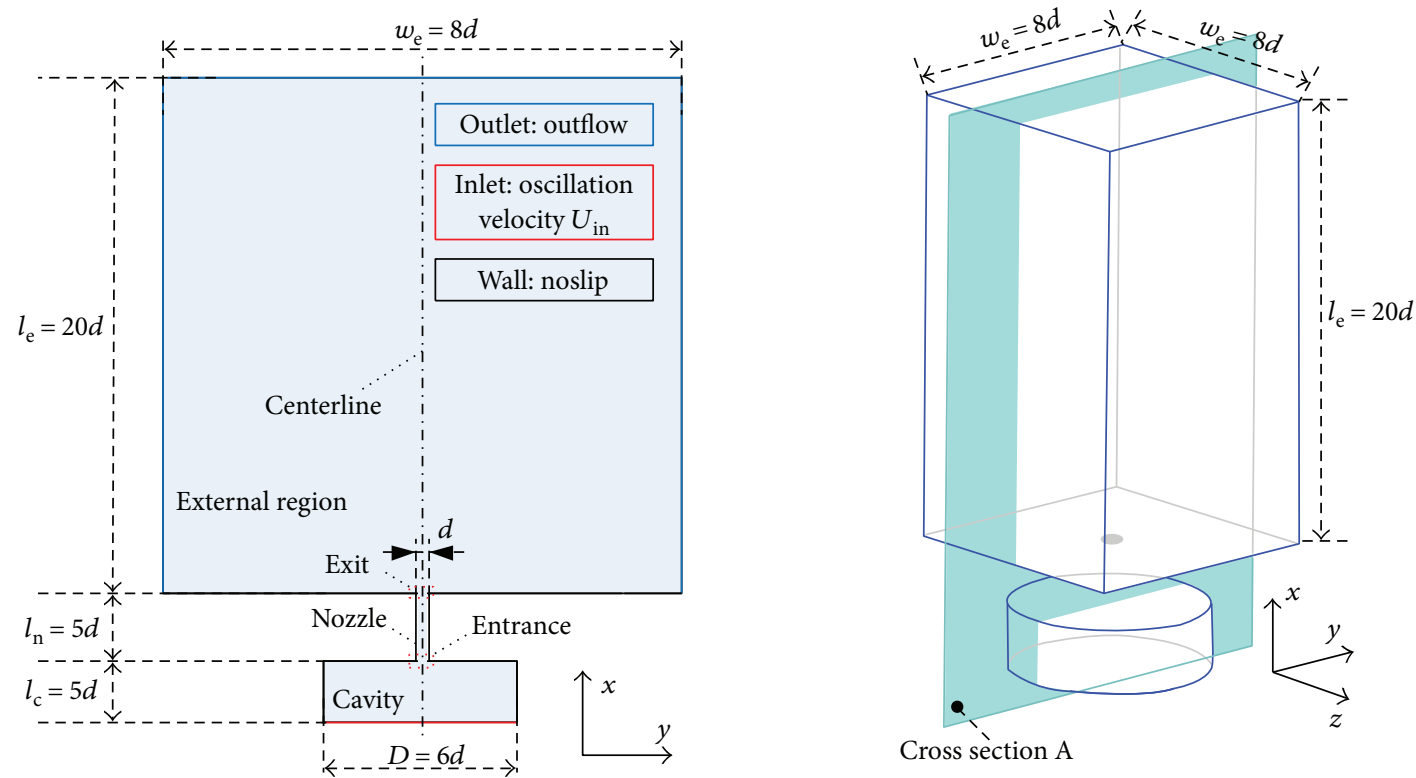

(b) 2-D and 3-D nondimensional model of synthetic jet cavity

Figure 3: Numerical model of synthetic jet cavity.

distributions of flux ratio $q$ through the diaphragm and nozzle exit are shown in Figure 4(b), where $\theta$ is the phase angle relative to the diaphragm velocity. The exit phase angle $\Phi=90^{\circ}$ corresponds to the maximum magnitude of expulsion velocity, while $\Phi=270^{\circ}$ corresponds to the maximum magnitude of ingestion velocity.

The flux and flux ratio were computed from

$$
\begin{aligned}
& Q(t)=\int_{0}^{A} U(t) \mathrm{d} s, \\
& q(t)=\frac{Q(t)}{Q_{\_ \text {max }}(t)},
\end{aligned}
$$

where $A$ is the area of the diaphragm or nozzle exit, $A_{\text {dia }}$ or $A_{\mathrm{n}} \cdot Q_{\max }$ is the maximum magnitude of the inlet or exit, $Q_{\text {dia_max }}$ or $Q_{\text {nozzle_exit_max. }}$

The flux ratios at the diaphragm and nozzle exit are defined as

$$
\begin{aligned}
q_{\text {dia }}(t) & =\frac{Q_{\text {dia }}(t)}{Q_{\text {dia_max }}(t)}, \\
q_{\text {nozzle_exit }}(t) & =\frac{Q_{\text {nozzle_exit }}(t)}{Q_{\text {nozzle_exit_max }}(t)} .
\end{aligned}
$$

Both of the diaphragm and nozzle exit fluxes have sinusoidal distribution features, within three working cycle example of lattice time from $39,600 \mathrm{lu}$ to $82,500 \mathrm{lu}(0.00792 \mathrm{~s}$ to $0.0165 \mathrm{~s}$ ). And the varying trend of the exit flux has the same cycle period as the one of the diaphragm flux, however, with a phase angle delay of about $180^{\circ}$. As seen in Figure 4(a), the flux magnitudes of the diaphragm and nozzle exit are different, however, the average values of the diaphragm flux and nozzle exit flux at each respective position during the analysis time are both essentially 0 , which means that the mass conservation can be met in one working cycle for the diaphragm and nozzle exit, respectively. And after the fifth cycle in Figure 4(b), the fluctuation amplitude of the flux ratio at the nozzle exit in the ingestion stroke is approximately the same as the expulsion, confirming zero-net mass flux for this simulation of the synthetic jet.

Figure 5 shows a typical example of the phase-averaged velocity cycle at the exit centerline. $U_{\mathrm{cl}}$ is the centerline jet velocity at the nozzle exit plane.

As seen in Figure 5, the phase-averaged exit velocity at the centerline also shows periodic variation, similar to the diaphragm velocity and flux. The velocity varies roughly sinusoidally; however, the maximum ingestion velocity (about $-4 \mathrm{~m} / \mathrm{s}$ ) does not have the same maximum magnitude 


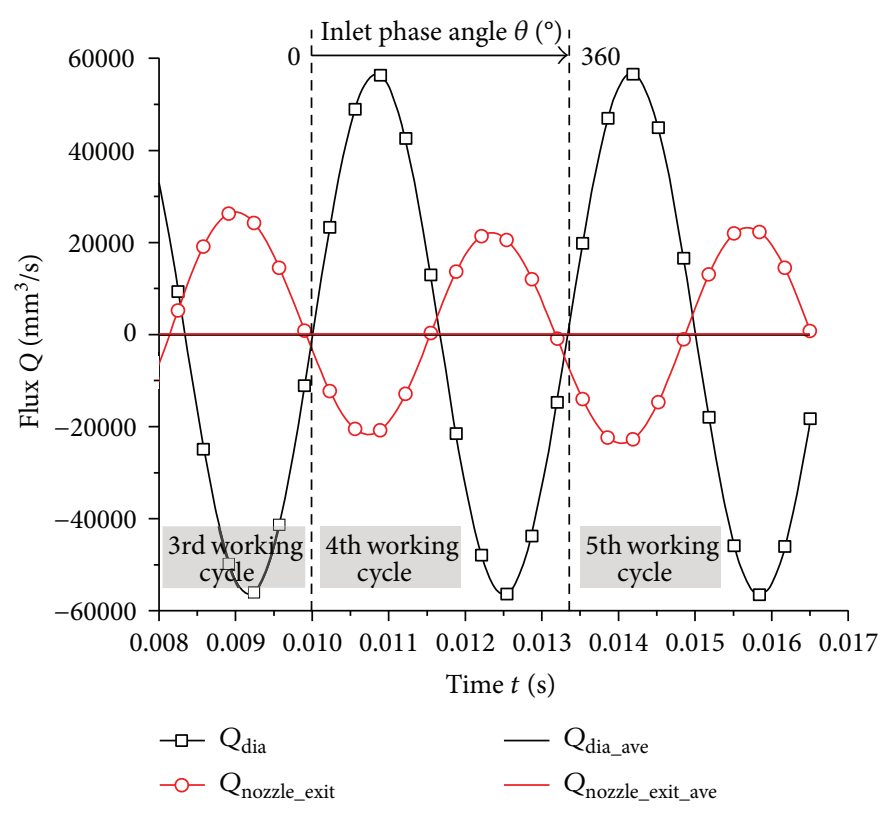

(a) Flux at the diaphragm and nozzle exit

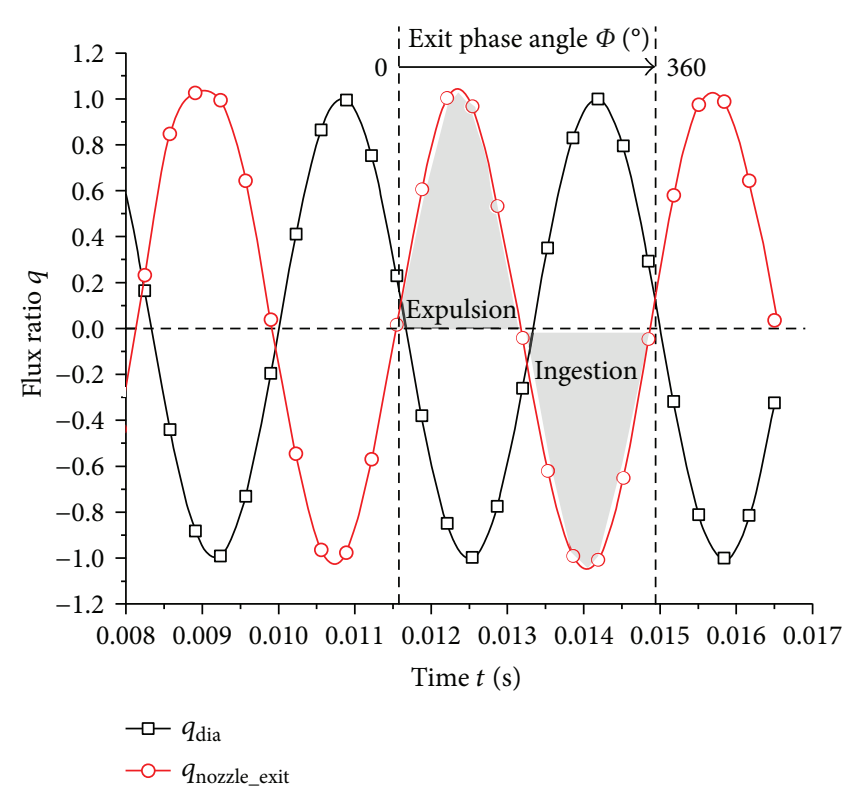

(b) Flux ratio at the diaphragm and nozzle exit

Figure 4: Physical flux and flux ratio at the diaphragm and nozzle exit from 3rd working cycle to 5th working cycle.

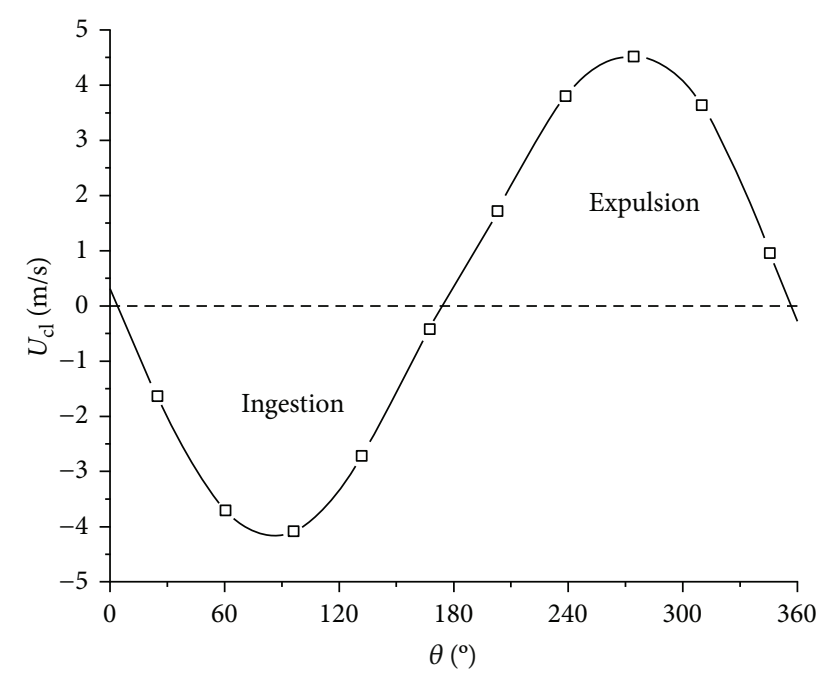

FIGURE 5: Velocity distribution at the nozzle exit centerline in the 4 th cycle.

as the expulsion velocity (about $4.5 \mathrm{~m} / \mathrm{s}$ ). A similar result also can be noticed in Feero et al. [14] that the maximum ingestion velocity at the centerline of the exit plane does not need to match the maximum expulsion velocity in order for the jet to have zero-net mass flux as the outgoing flow moves through the centerline of the nozzle in the expulsion and the ingestion velocity occurs primarily along the periphery.

Figure 6 shows the axial velocity distribution at the nozzle exit line at different times throughout the cycle. The origin of the cylindrical coordinate system is the center of the nozzle exit plane, and the radial location of these velocity data across the nozzle is shown in Figure 7 for $l / d$ between -0.5 and 0.5 .

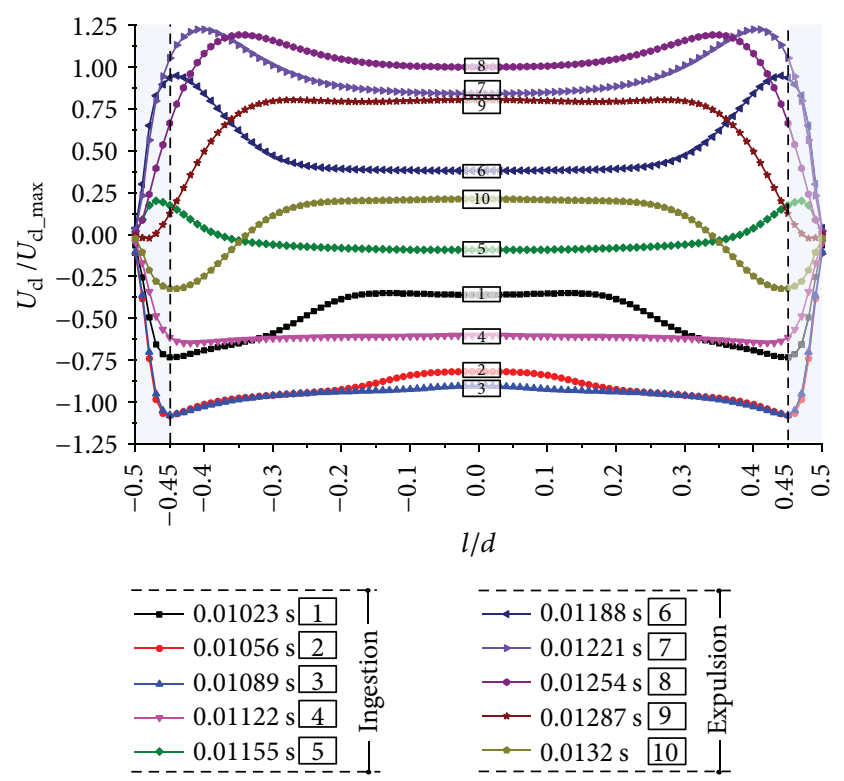

FIgURe 6: Radial profile of normalized jet velocity at the nozzle exit line in the 4th cycle.

The axial velocity (in the $x$-direction) at $l / d$ of -0.5 and 0.5 is zero. From $\theta=0$ to $180^{\circ}$ (physical times from $t=0.01023 \mathrm{~s}$ to $0.01155 \mathrm{~s}$ ), the velocities are in the negative $x$-direction, the ingestion portion of the cycle, while from $\theta=180^{\circ}$ to $360^{\circ}$ (from $0.01188 \mathrm{~s}$ to $0.0132 \mathrm{~s}$ ), the velocities are positive for the expulsion stroke. As shown in Figure 6, the majority of velocity profiles are roughly uniform throughout the center region with local variations near the wall. It can be seen in Figure 6 that the velocity magnitude near the wall $(|l / d|=0.45$ to 0.5$)$ in the ingestion stroke is 

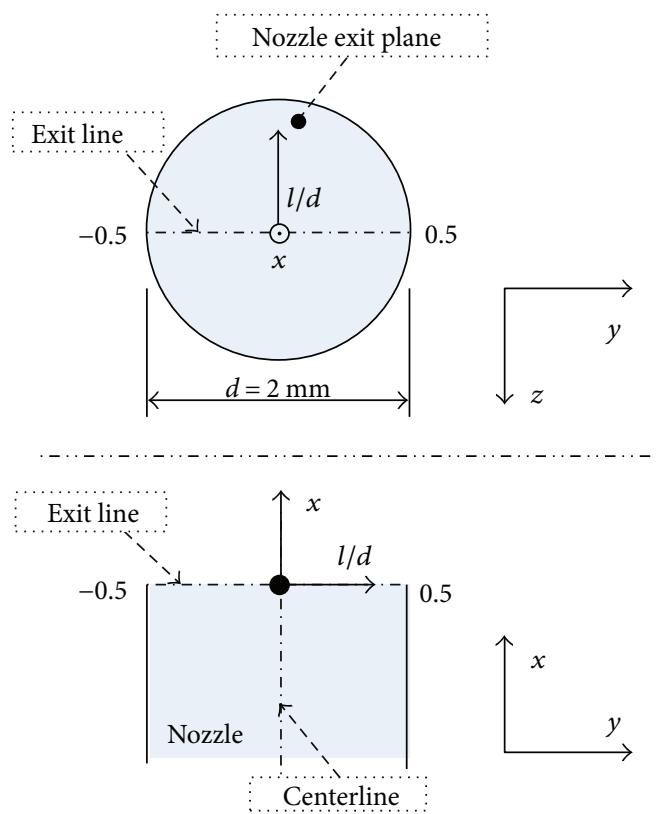

FIGURE 7: Radial location of simulated jet velocity across the nozzle exit plane.

obviously higher than the one in the expulsion part. The maximum velocity of each ingestion profile is closer to the nozzle exit wall, and the magnitude of the centerline velocity in the expulsion stroke is larger than that for ingestion. Expulsion occurs mainly through the nozzle centerline; however, the ingestion occurs primarily along the periphery.

Figure 8 compares the normalized axial velocity at the nozzle exit line between the experiment and present simulation. The maximum magnitudes of expulsion velocity (exit phase angle $\left.\Phi=90^{\circ}\right)$ and ingestion velocity $\left(\Phi=270^{\circ}\right)$ were extracted and compared.

As observed in Figure 8, the numerical results are in good agreement with Feero et al. [14], particularly for the case of $\Phi=90^{\circ}$ where the flow is primarily axial. The average error at $\Phi=90^{\circ}$ is about $5.6 \%$ while for $\Phi=270^{\circ}$, it is approximately $8.1 \%$. It should be noted that the measurements were performed with hotwire anemometry and thus were unable to resolve the reverse flow or direction. The small peaks in the velocity profiles can be observed near the wall of the nozzle for both curves. As the flow reverses at the nozzle exit center due to the cavity inlet velocity, the velocity remains highest near the wall of the nozzle.

A sample of velocity contours at different times during the 4 th cycle is seen in Figure 9 and corresponds to the data presented in Figure 5.

Figures 9(a)-9(e) are for the ingestion portion of the cycle, while Figures $9(\mathrm{f})-9(\mathrm{j})$ are the expulsion stroke. Figure 9 also illustrates the vortex motion outside the synthetic jet actuator and within the cavity at different phase angles over one working cycle, exhibiting a clear jet formation. The same phase difference between the diaphragm velocity and nozzle exit velocity (seen in Figure 4) is clearly seen. From Figures 9(a)-9(e), vortex el moves upward and vortex cl moves downward into the cavity. This is then

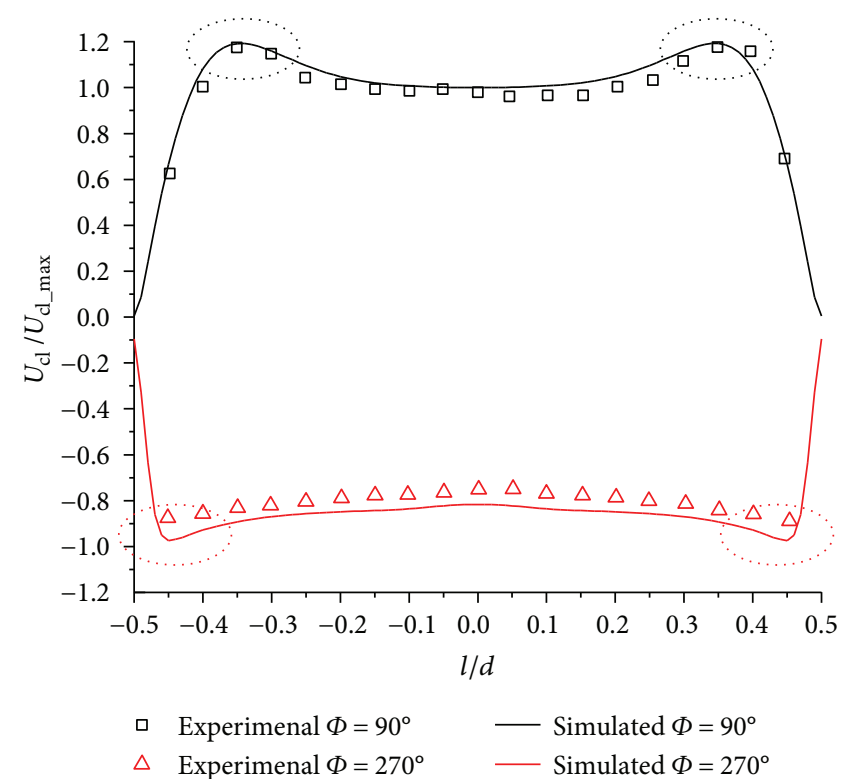

Figure 8: Comparison of normalized velocity of the exit line at different exit phase angles between the experiment and the simulation.

followed by the new vortices e 2 and $\mathrm{c} 2$ that generate and convect upstream (c2) or downstream (e2) (Figure 9(f)-9(j)).

$\theta=18^{\circ}$ (Figure 9(a)) was chosen to correspond to the commencement of the upward motion of the diaphragm modeled here by the oscillatory velocity boundary condition at the bottom of the cavity. It coincides with the start of the ingestion stroke of ambient air into the cavity. Figure 9(a) shows some remnants of the previous vortex pairs el and $\mathrm{cl}$ in the external region and cavity, caused by the previous expulsion and ingestion of the synthetic jet. It also shows the presence of corner vortices at the nozzle entrance. At $\theta=54^{\circ}$ (Figure 9(b)), the new vortex pair c2 rolls up at the entrance of the nozzle and its size is of the order of the nozzle width. Separation of the shear layer near the nozzle exit is also readily visible. When the roll-up process is completed at the maximum-ingestion phase of $90^{\circ}$ as illustrated (Figure $9(\mathrm{c})$ ), the mixing of the primary vortex pair is mostly completed. Then with the upward motion of the diaphragm, the ingestion velocity in the nozzle decreases gradually at $\theta=126^{\circ}$ (Figure $9(\mathrm{~d})$ ) and $162^{\circ}$ (Figure $9(\mathrm{e})$ ). The vortex pair inside the cavity starts to grow in size while it descends into the jet cavity, and the ingestion phase is completed.

The expulsion phase has commenced at $\theta=198^{\circ}$ as shown in Figure 9(f) which corresponds to the initiation of the downward motion of the diaphragm. At $\theta=234^{\circ}$ (Figure 9(e)), the shear layer separation occurs near the nozzle entrance, and expulsion begins the formation of a vortex pair at the nozzle exit. At the maximum-expulsion phase of $270^{\circ}$ (Figure $9(\mathrm{~h})$ ), the vortex pair detaches from the nozzle exit plane and grows in size as it advects downstream. After $\theta=342^{\circ}$ (Figure 9(i)), the expulsion phase completes and the ingestion stroke commences, by which time the vortex pair has advected sufficiently downstream that it is not 


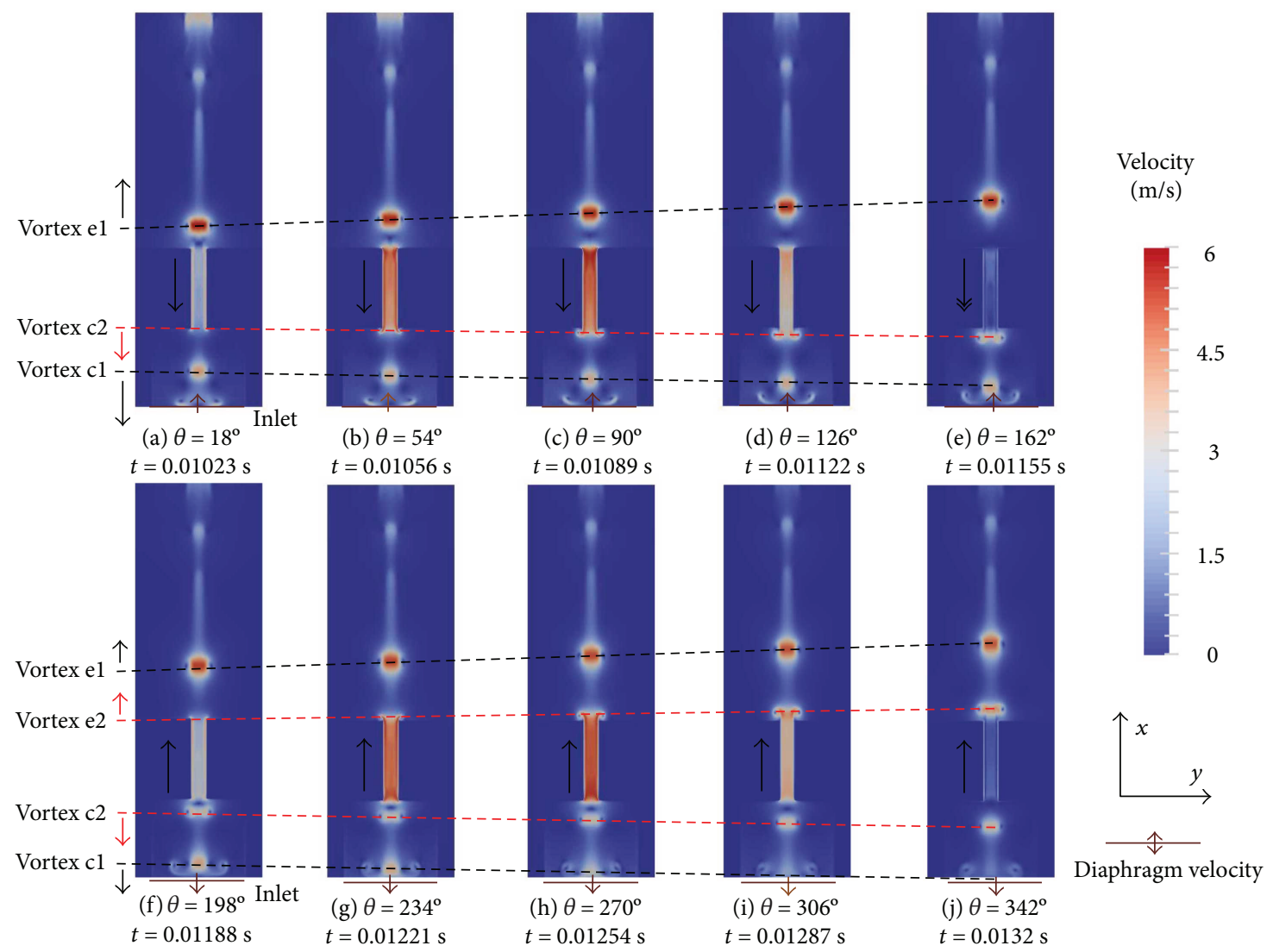

Figure 9: Velocity contour in the 4th cycle at cross section A with sinusoidal diaphragm velocity condition.

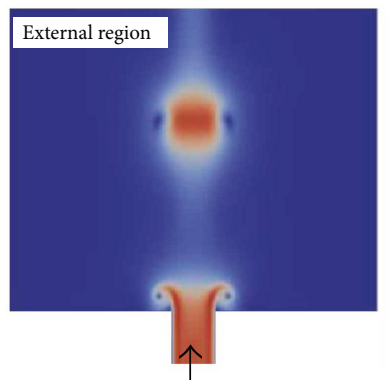

(1) External region

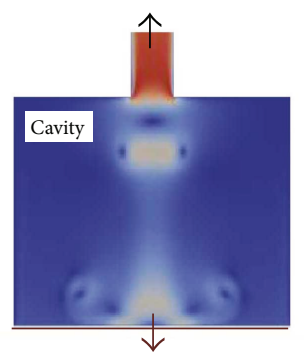

(2) Cavity

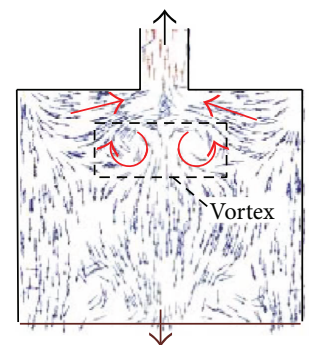

(a) Expulsion stroke $\Phi=90^{\circ} / \theta=270^{\circ}$
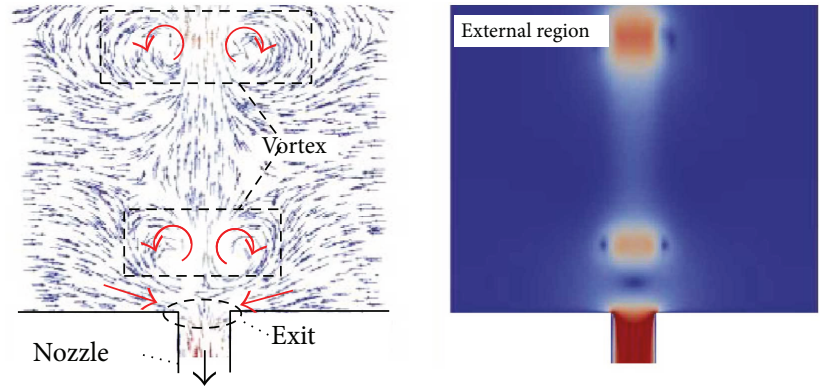

(1) External region
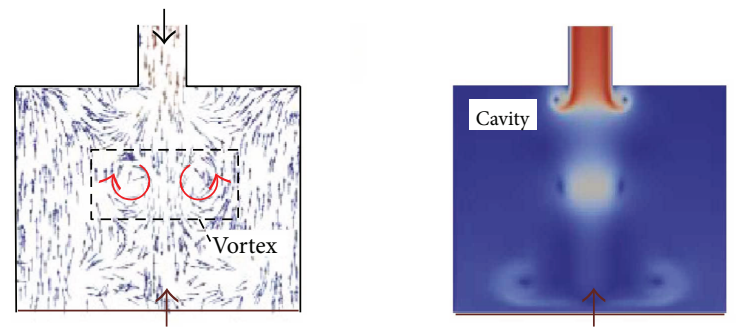

(2) Cavity

(b) Ingestion stroke $\Phi=270^{\circ} / \theta=90^{\circ}$

Figure 10: Vector distribution of phase-averaged velocity at cross section A at $\Phi=90^{\circ}$ and $270^{\circ}$.

affected by the suction of ambient fluid into the cavity. The flow in the cylindrical cavity and the outer flow will cause a phase difference between the diaphragm velocity and nozzle exit velocity, and the value of the phase difference is determined by the oscillating amplitude, oscillating frequency, cavity volume, and cavity shape [23]. 
Figure 10 shows the vector distribution of the phaseaveraged velocity and vortex pairs in the external region and the cavity at exit phase angle $\Phi=90^{\circ}$ and $270^{\circ}$ at cross section A, corresponding to the velocity contour in Figure 8. When $\Phi$ is set as $90^{\circ}$, fluid flows out from the nozzle, and the synthetic jet cavity is in the expulsion stroke with maximum velocity. The flow leaving the cavity will be in counter flow with the decelerating vortex pair from the ingestion stroke and there is sufficient room in the upper cylindrical cavity for the outgoing flow to move around these vortices (Figure 10(a)). At $\Phi=270^{\circ}$, the fluid flows into the nozzle, and the magnitude of the ingestion velocity is maximum. The vortex pair generated during expulsion is far enough from the nozzle exit, so the peripheral fluid is ingested into the nozzle. A vortex pair is generated near the wall of the nozzle exit at $\Phi=90^{\circ}$ in the expulsion stroke, and the axial vortex velocity near the wall is higher than the centerline velocity at the nozzle exit. At $\Phi=90^{\circ}$, the velocity profile in Figure 8 is affected by the presence of these vortices. For $\Phi=270^{\circ}$ as well, fluid flows into the cavity and the vortex pair occurs near the wall of the nozzle entrance. We can see that the axial velocity near the wall is again higher than the centerline velocity at the nozzle entrance. The upward diaphragm velocity also has larger influence on the backward centerline velocity at the nozzle entrance.

\section{Conclusion}

A numerical simulation of synthetic jet was conducted with the 3-D lattice Boltzmann method. An unsteady synthetic jet simulation was carried out. In comparison to experimental results, the 3-D LBM accurately represented the expected flow feature while also providing insight into the ingestion/expulsion mechanics. The computed results showed that the ingestion and expulsion strokes could be observed and identified by the velocity profiles and velocity vectors in one working cycle of the synthetic jet. The expected zero-net mass-flux behavior was confirmed, providing further confidence in the previous measured velocity profiles. The ingestion flow was found to occur along the periphery of the jet and the expulsion flow along the centerline. Using the 3-D LBM and appropriate driving boundary condition, a clear synthetic jet formation and evolution of vortex could be obtained and analyzed, which is important to develop strategies for active flow control.

\section{Conflicts of Interest}

The authors declare that they have no conflicts of interest.

\section{Acknowledgments}

The authors gratefully acknowledge the support of the Natural Sciences and Engineering Research Council (NSERC) and the Southern Ontario Smart Computing Innovation Platform (SOSCIP). Computations were performed on the Sandybridge (Sandy) cluster at the SciNet HPC Consortium which includes support from the Canada Foundation for Innovation under the auspices of Compute
Canada, the Government of Ontario, Ontario Research Fund-Research Excellence, the University of Toronto, and the China Scholarship Council (CSC).

\section{References}

[1] D. Greenblatt, K. B. Paschal, Y. Chung-Sheng, and J. Harris, "Experimental investigation of separation control part 2: zero mass-flux oscillatory blowing," AIAA Journal, vol. 44, no. 12, pp. 2831-2845, 2006.

[2] N. Ramos-Pedroza, W. MacKunis, and M. Reyhanoglu, "A sliding mode LCO regulation strategy for dual-parallel underactuated UAV systems using synthetic jet actuators," International Journal of Aerospace Engineering, vol. 2015, Article ID 795348, 7 pages, 2015.

[3] M. A. Feero, P. Lavoie, and P. E. Sullivan, "Influence of synthetic jet location on active control of an airfoil at low Reynolds number," Experiments in Fluids, vol. 58, no. 8, 2017.

[4] A. Pavlova and M. Amitay, "Electronic cooling using synthetic jet impingement," Journal of Heat Transfer, vol. 128, no. 9, p. 897, 2006.

[5] D. S. Kercher, J. B. Lee, O. Brand, M. G. Allen, and A. Glezer, "Microjet cooling devices for thermal management of electronics," IEEE Transactions on Components and Packaging Technologies, vol. 26, no. 2, pp. 359-366, 2003.

[6] M. Amitay, D. R. Smith, V. Kibens, D. E. Parekh, and A. Glezer, "Aerodynamic flow control over an unconventional airfoil using synthetic jet actuators," AIAA Journal, vol. 39, no. 3, pp. 361-370, 2001.

[7] A. Glezer and M. Amitay, "Synthetic jets," Annual Review of Fluid Mechanics, vol. 34, no. 1, pp. 503-529, 2002.

[8] C. Lee, G. Hong, Q. P. Ha, and S. G. Mallinson, "A piezoelectrically actuated micro synthetic jet for active flow control," Sensors and Actuators, A: Physical, vol. 108, no. 1-3, pp. 168-174, 2003.

[9] C. Chovet, M. Lippert, L. Keirsbulck, and J.-M. Foucaut, "Dynamic characterization of piezoelectric micro-blowers for separation flow control," Sensors and Actuators A: Physical, vol. 249, pp. 122-130, 2016.

[10] S. G. Sawant, B. George, L. S. Ukeiley, and D. P. Arnold, "Microfabricated electrodynamic synthetic jet actuators," Journal of Microelectromechanical Systems, vol. 27, no. 1, pp. 95-105, 2017.

[11] K. Okada, A. Oyama, K. Fujii, and K. Miyaji, "Computational study on effect of synthetic jet design parameters," International Journal of Aerospace Engineering, vol. 2010, Article ID 364859, 11 pages, 2010.

[12] X. Ma, H. Guo, Z. Fan, and L. Zhang, "Investigating of simulation methods for synthetic jet," Procedia Engineering, vol. 31, pp. 416-421, 2012.

[13] Y. Utturkar, R. Mittal, P. Rampunggoon, and L. Cattafesta, "Sensitivity of synthetic jets to the design of the jet cavity," in 40th AIAA Aerospace Sciences Meeting \& Exhibit, Reno, NV, USA, 2002.

[14] M. A. Feero, P. Lavoie, and P. E. Sullivan, "Influence of cavity shape on synthetic jet performance," Sensors and Actuators A: Physical, vol. 223, pp. 1-10, 2015.

[15] D. A. Perumal and A. K. Dass, "Simulation of incompressible flows in two-sided lid-driven square cavities. Part II-LBM," CFD Letters, vol. 2, no. 1, pp. 25-38, 2010. 
[16] D. A. Perumal and A. K. Dass, "Multiplicity of steady solutions in two-dimensional lid-driven cavity flows by Lattice Boltzmann Method," Computers \& Mathematics with Applications, vol. 61, no. 12, pp. 3711-3721, 2011.

[17] C. Wang, H. Tang, F. Duan, and S. C. M. Yu, "Control of wakes and vortex-induced vibrations of a single circular cylinder using synthetic jets," Journal of Fluids and Structures, vol. 60, pp. 160-179, 2016.

[18] T. Mautner, "Application of the synthetic jet concept to low Reynolds number biosensor microfluidic flows for enhanced mixing: a numerical study using the lattice Boltzmann method," Biosensors \& Bioelectronics, vol. 19, no. 11, pp. 1409-1419, 2004.

[19] G. Fu, B. A. Younis, L. Sun, and S. Dai, "Vortex shedding control using jets: a computational study with lattice Boltzmann method," in ASME 2016 35th International Conference on Ocean, Offshore and Arctic Engineering, Busan, South Korea, 2016.

[20] K. Iglberger, N. Thürey, and U. Rüde, "Simulation of moving particles in 3D with the Lattice Boltzmann method," Computers \& Mathematics with Applications, vol. 55, no. 7, pp. 1461-1468, 2008.

[21] R. Holman, Y. Utturkar, R. Mittal, B. L. Smith, and L. Cattafesta, "Formation criterion for synthetic jets," AIAA Journal, vol. 43, no. 10, pp. 2110-2116, 2005.

[22] R. B. Kotapati, R. Mittal, and L. N. Cattafesta III, "Numerical study of a transitional synthetic jet in quiescent external flow," Journal of Fluid Mechanics, vol. 581, pp. 287-321, 2007.

[23] M. Jain, B. Puranik, and A. Agrawal, "A numerical investigation of effects of cavity and orifice parameters on the characteristics of a synthetic jet flow," Sensors and Actuators A: Physical, vol. 165, no. 2, pp. 351-366, 2011. 


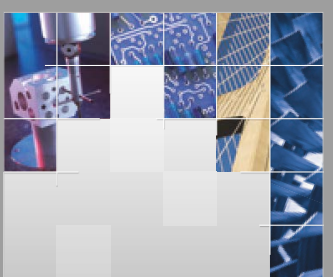

\section{Enfincering}
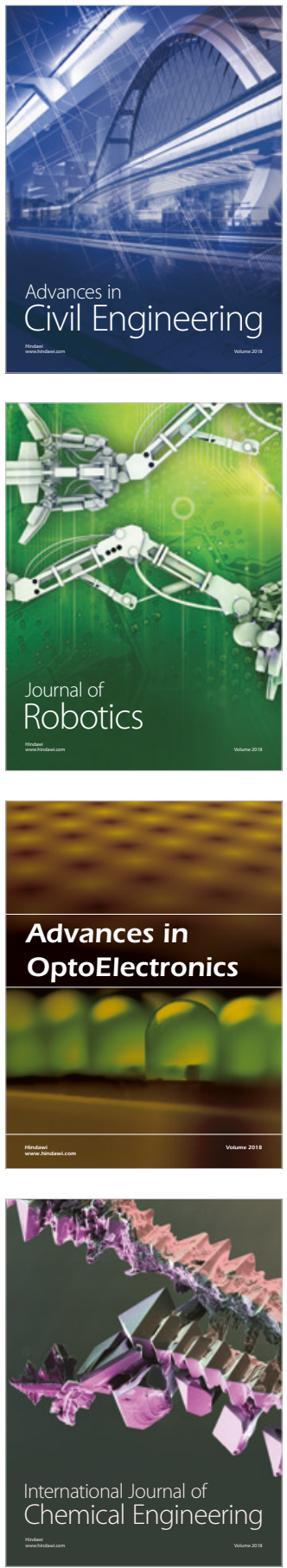

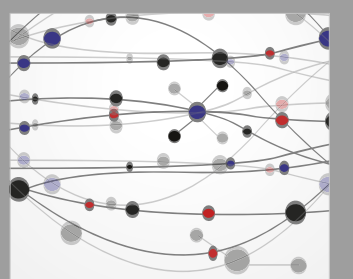

\section{Rotating \\ Machinery}

The Scientific World Journal

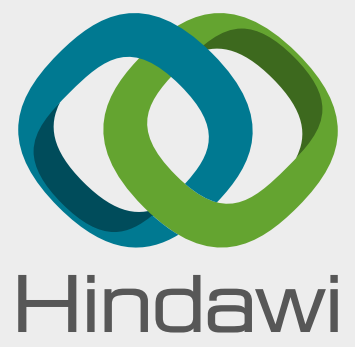

Submit your manuscripts at

www.hindawi.com
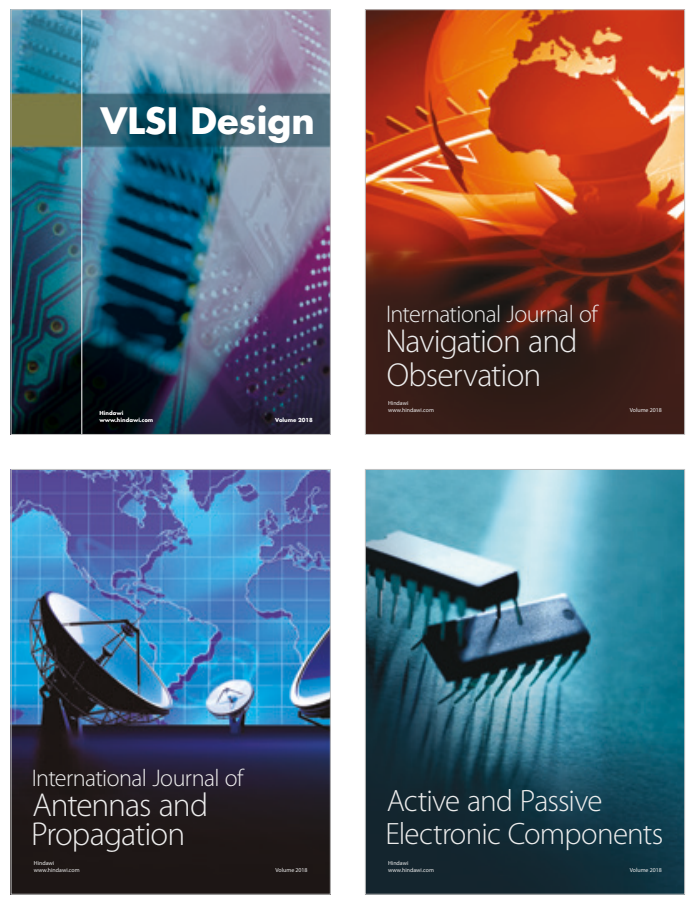
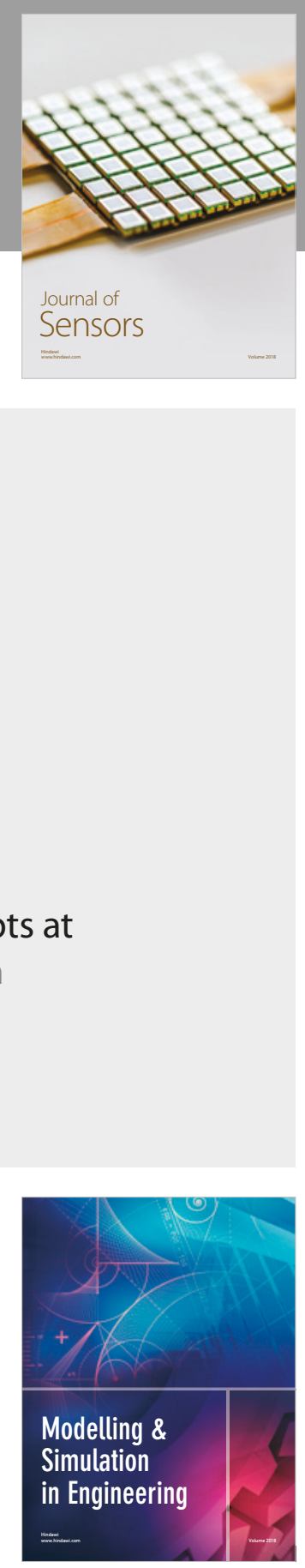

\section{Advances \\ Multimedia}
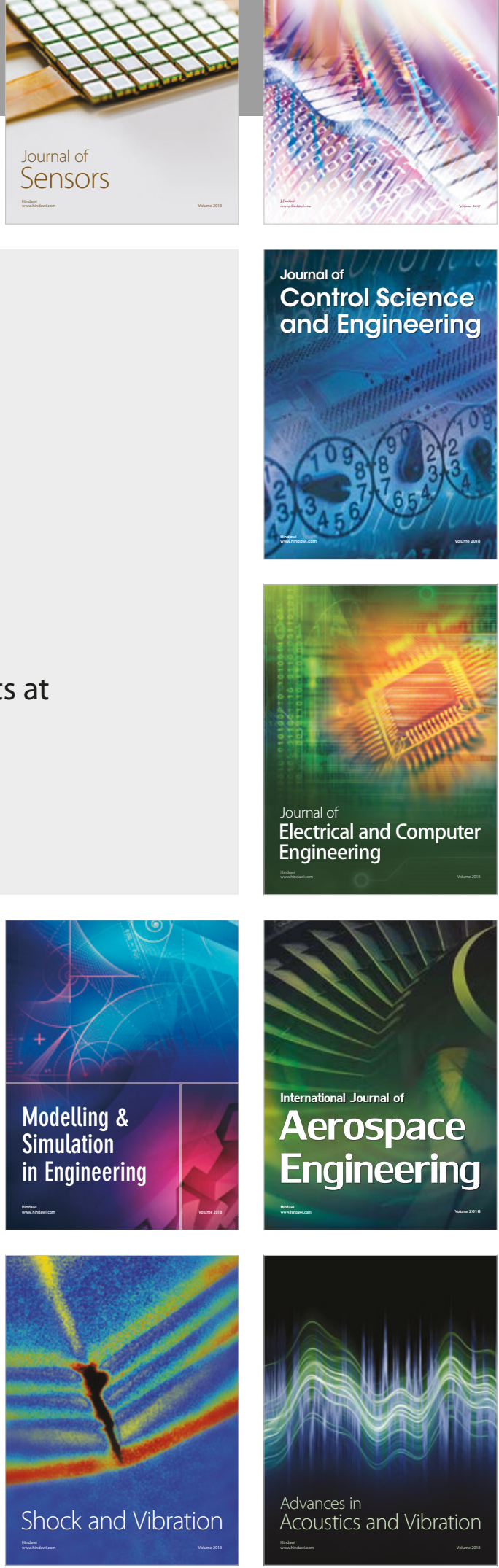